\title{
Technical factors that minimize the occurrence of early graft failure in pancreas transplantation
}

\author{
Byunghyun Choi, Jeho Ryu, Kwangho Yang, Taebeom Lee, Jaeryong Shim, Hyojung Ko
}

Department of Surgery-Transplantation, Pusan National University Yangsan Hospital, Busan, Korea

Background: Pancreas transplantation is the only treatment in insulin-dependent diabetes that can result in long-term euglycemia without external insulin. However, pancreatic transplant has become debatable following the improvements in the results of islet transplantation and artificial pancreas, which are evolving rapidly. Therefore, surgeons who perform pancreas transplant require the best surgical technique that can minimize technical failure in such transplantations. This study aimed to report our experiences with pancreatic transplantations.

Methods: We transplanted 65 pancreatic grafts between 2015 and 2020 (simultaneous pancreas and kidney transplant, n=12; pancreas transplant after kidney, $n=16$; pancreas transplant alone, $n=37$ ). Except for one death that occurred due to hypoxic brain damage immediately after the surgery, we did not observe postoperative technical failure. At our center, we commonly performed duodeno-duodenal anastomosis in the retroperitoneal space.

Results: There was no incidence of leakage from the duodenum even after immunologic graft failure. In order to prevent venous thrombosis, which is the most common cause of technical failure, we always used the inferior vena cava for anastomosis and added a special procedure to the bench procedure; subsequently, there were no cases of technical failure due to thrombosis post-transplantation. Therefore, the 1-year graft survival (insulin-free) rate was more than 95\%.

Conclusions: Islet transplantation and artificial pancreas still cannot be expected to be as successful as pancreatic transplantation. Therefore, improving the surgical technique will maintain pancreatic transplantation as the best treatment in insulin-dependent diabetes.

Corresponding author: Byunghyun Choi

E-mail:gmoolpop@gmail.com

(c) The Korean Society for Transplantation

This is an Open Access article distributed under the terms of the Creative Commons Attribution Non-Commercial License (http://creativecommons.org/licenses/by-nc/4.0/) which permits unrestricted non-commercial use, distribution, and reproduction in any medium, provided the original work is properly cited. 